\section{Un enfoque digital para restaurar un diente anterior traumatizado durante la pandemia COVID-19: reporte del caso}

\section{A digital approach to restore a traumatized anterior tooth during the COVID-19 pandemic: A clinical report}

\section{Resumen}

Los profesionales de la salud oral tienen un mayor riesgo de infección con el nuevo coronavirus 2019 (COVID-19), debido a la comunicación cercana a la cavidad oral y la exposición a saliva, sangre y otros fluidos corporales. Los enfoques digitales pueden reducir el tiempo clínico y contribuir a limitar el riesgo de infección. El objetivo del presente trabajo es presentar un enfoque digital para restaurar un diente anterior traumatizado, durante la pandemia de COVID-19. Paciente sexo feminino de 28 ańos, con movilidad anormal de la pieza $1.1 \mathrm{y}$ antecedente de traumatismo dental en la adolescencia. Se realizó una evaluación inicial para detectar signos y síntomas de una posible infección por COVID-19. Las imágenes de la tomografía computarizada mostraron fractura coronaria y presencia de reabsorción cervical externa en el incisivo central derecho. El plan de tratamiento consistió en la rehabilitación del diente fracturado a través de una restauración retenida con un poste de fibra de vidrio, usando un abordaje digital por medio de un escáner intraoral con diseño y fabricación asistido por computadora (CAD/CAM). Los enfoques digitales en odontología podrían contribuir a reducir el riesgo de infección por COVID-19 en pacientes y dentistas durante los tratamientos restauradores.

Palabras clave: SARS-CoV; COVID-19; Odontología; Consultorio dental; Estética dental (fuente: DeCS BIREME).

\begin{abstract}
Dental professionals are at increased risk of infection with the new coronavirus 2019 (COVID-19) due to face-to-face communication and exposure to saliva, blood and other body fluids. Digital approaches can reduce clinical time and contribute to limiting the risk of infection. The objective of this work was to report a digital approach to restoring a traumatized anterior tooth during the COVID-19 pandemic. A 28-year-old female with abnormal mobility in the crown of an upper anterior tooth with history of dental trauma in the adolescence. An initial screening was performed to check for signs and symptoms of possible COVID-19 infection. Cone Beam Computed Tomography images showed a crown fracture and the presence of external cervical resorption in the right permanent central incisor. The treatment plan consisted of rehabilitation of the fractured tooth through a restoration retained with a fiberglass post with a digital approach using an intraoral scanner, and computer-aided design and fabrication - CAD/CAM. Digital approaches in dentistry
\end{abstract}

\section{Caso Clínico}

Luiz Gustavo Fernandes Lima Oliveira ${ }^{1, a}$, Alicia Rocha Costa ${ }^{2, b}$, Marina Pontes do Nascimento ${ }^{2, a}$, Marlus da Silva Pedrosa ${ }^{3,4, a}$

${ }^{1}$ Consultorio Odontológico Luiz Gustavo Odontologia Estética, Teresina, Piauí, Brasil.

${ }^{2}$ Centro Universitario UNINOVAFAPI, Teresina, Piauí, Brasil.

${ }^{3}$ Consultorio Marina Pontes do Nascimento, Teresina, Piauí, Brasil.

${ }^{4}$ Universidade de São Paulo, Faculdade de Odontologia, Departamento de Biomateriales y Biología Oral, São

Paulo, Brasil.

${ }^{a}$ Cirujano Dentista.

${ }^{\mathrm{b}}$ Estudiante de Odontología.

\section{Correspondencia:}

Marlus da Silva Pedrosa: marluspedrosa@gmail.com Av. Prof. Lineu Prestes, 2227 - Ciudad Universitaria, São Paulo - SP - Brasil

ORCID: 0000-0002-4052-7208

\section{Coautores:}

Luiz Gustavo Fernandes Lima Oliveira: luizgustavocd@ hotmail.com

ORCID: 0000-0003-0839-0317

Alicia Rocha Costa: aliciaantoni@gmail.com

ORCID: 0000-0002-5475-3388

Marina Pontes do Nascimento: marinapnascimento@

yahoo.com.br

ORCID: 0000-0002-9623-130X

\section{Editora:}

Rosse Mary Falcón-Antenucci

Universidad Inca Garcilaso de la Vega, Perú

Conflicto de intereses: los autores declaran no tener conflictos de interés.

Fuente de financiamiento: autofinanciado

Recibido: 06/09/21

Aceptado: $23 / 09 / 21$

Publicado: 21/01/22

(C) Los autores. Este artículo es publicado por la revista Odontología Sanmarquina de la Facultad de Odontología, Universidad Nacional Mayor de San Marcos. Este es un artículo de acceso abierto, distribuido bajo los términos de la licencia Creative Commons Atribución 4.0 Internacional (CC BY 4.0) [https://creativecommons.org/ licenses/by/4.0/deed.es] que permite el uso, distribución y reproducción en cualquier medio, siempre que la obra original sea debidamente citada de su fuente original. 
including intraoral scanners and CAD/CAM could contribute to reducing the risk of COVID-19 infection in patients and dentists during restorative treatments.

Keywords: SARS virus; Coronavirus infections; Dentistry; Dental offices; Dental esthetics Operative dentistry (source: MeSH NLM).

\section{Introducción}

La nueva enfermedad por coronavirus 2019 (COVID-19) causada por el síndrome respiratorio agudo severo coronavirus 2 (SARS-CoV-2) es la última enfermedad infecciosa que se propagó rápidamente en todo el mundo ${ }^{1}$. La Organización Mundial de la Salud (OMS) declaró a la COVID-19 una emergencia de salud pública de interés internacional ${ }^{2}$.

Aunque actualmente hay vacunas disponibles, la mejor estrategia para tratar el SARS-CoV-2 incluye controlar las fuentes de infección ${ }^{3}$. La transmisión de persona a persona consiste en la transmisión indirecta al toser, estornudar, la transmisión por inhalación de micropartículas de aerosol y el contacto directo con las membranas mucosas bucales, nasales y oculares ${ }^{4}$.

La práctica odontológica es responsable de un mayor riesgo de infección por SARS-CoV-2 debido a la comunicación cara a cara y la exposición a saliva, sangre y otros fluidos corporales ${ }^{2}$. Por lo tanto, cada paciente debe ser considerado como potencialmente infectado y los dentistas deben revisar sus políticas de control de infecciones y las medidas preventivas para brindar atención dental y mitigar la propagación de la infección ${ }^{5}$.

Se han publicado en todo el mundo varias pautas para el cuidado odontológico durante la pandemia de COVID-19 ${ }^{5-9}$. El Consejo Federal de Odontología y la Agencia Reguladora de Salud de Brasil recomiendan que la atención dental se brinde solo en casos de urgencia y emergencia, siguiendo estrictas pautas de control de infecciones ${ }^{10-12}$. Las emergencias dentales son procedimientos que requieren intervención para aliviar las molestias. Esto también incluye traumatismos dentales, caries extensas o restauraciones defectuosas ${ }^{11-14}$.

Las tecnologías digitales son alternativas fiables a las técnicas convencionales para la fabricación de prótesis parciales fijas soportadas por dientes o implantes ${ }^{15}$. Los flujos de trabajo digitales tienen varias ventajas en comparación con los convencionales ${ }^{16,17}$. El tiempo total en la cita odontológica es significativamente menor para los flujos de trabajo digitales, ya que reducen los procedimientos clínicos y de laboratorio ${ }^{18-20}$. En la pandemia de COVID-19, siempre que sea posible, se deben implementar enfoques digitales en la práctica dental para reducir el tiempo de trabajo y así limitar el riesgo de infección ${ }^{21}$. En este manuscrito, presentamos un enfoque digital para restaurar un diente anterior traumatizado durante la pandemia de COVID-19.

\section{Reporte del caso}

Paciente de sexo feminino de 28 ańos de edad se presentó en el consultorio odontológico refiriendo movilidad anormal en la corona de un diente anterior superior. El paciente tuvo un trauma dental en su adolescencia (16 años). Y se le hizo un tratamiento de conducto radicular. El diente se oscureció con el tiempo y se realizaron siete intentos de blanqueamiento dental en diente no vital.

En la evaluación inicial para detectar signos y síntomas de una posible infección por COVID-19 ${ }^{5-7}$, el paciente no refirió fiebre, problemas respiratorios y contacto con casos confirmados o sospechosos de COVID-19. No hubo signos y síntomas de COVID-19, ni de trastornos sistémicos el día de la consulta.

Al examen clínico intraoral se notó una intensa decoloración (Figura 1) y movilidad de la corona del incisivo central derecho. Para confirmar la posibilidad de fractura coronaria, fue realizada una tomografía computarizada con haz cónico - CBCT (OP300 3D, Instrumentarium, EE.UU.), al analizar las imágenes, se observó fractura de la corona y presencia de reabsorción cervical externa (Figura 2) en el incisivo central derecho.

El plan de tratamiento consistió en la rehabilitación del diente fracturado mediante una restauración retenida con un poste de fibra de vidrio. El paciente firmó un formulario de consentimiento informado para el plan de tratamiento.

Para la fabricación de la restauración provisional se utilizó un abordaje digital con un escáner intraoral (3Shape TRIOS ${ }^{\circledR}$ ), además de diseño y fabricación asistidos por computadora - CAD/CAM (fresadora Zirkonzahn M1). La tecnología CAD-CAM se utilizó para compensar las deficiencias materiales relacionadas con el tiempo de los métodos tradicionales ${ }^{22}$.

La corona del diente fracturado se escaneó mientras aún estaba en la boca para reproducir su forma y textura en la corona provisional (Figura 1). Se preparó el conducto radicular para recibir el poste de fibra de vidrio ${ }^{23,24}$. La estructura dentaria restante (Figura 3) se preparó de acuerdo con el concepto de la técnica de preparación biológicamente orientada (BOPT) (Figura 4) ${ }^{25}$. Se fabricó e instaló un poste anatómicamente personalizado en el conducto radicular (Figura 5). Esta técnica consiste en personalizar un poste prefabricado de fibra de vidrio moldeando el conducto radicular con aplicación directa de resina compuesta ${ }^{26}$. Luego diseñamos la corona del diente usando una copia digital del diente original escaneado previamente (Figura 5). Se generó y fresó un archivo STL en polimetilmetacrilato multicolor (PMMA) para CAD/CAM. La figura 6, tomada por la propia paciente, muestra el resultado obtenido después de la cementación de la corona provisional. 


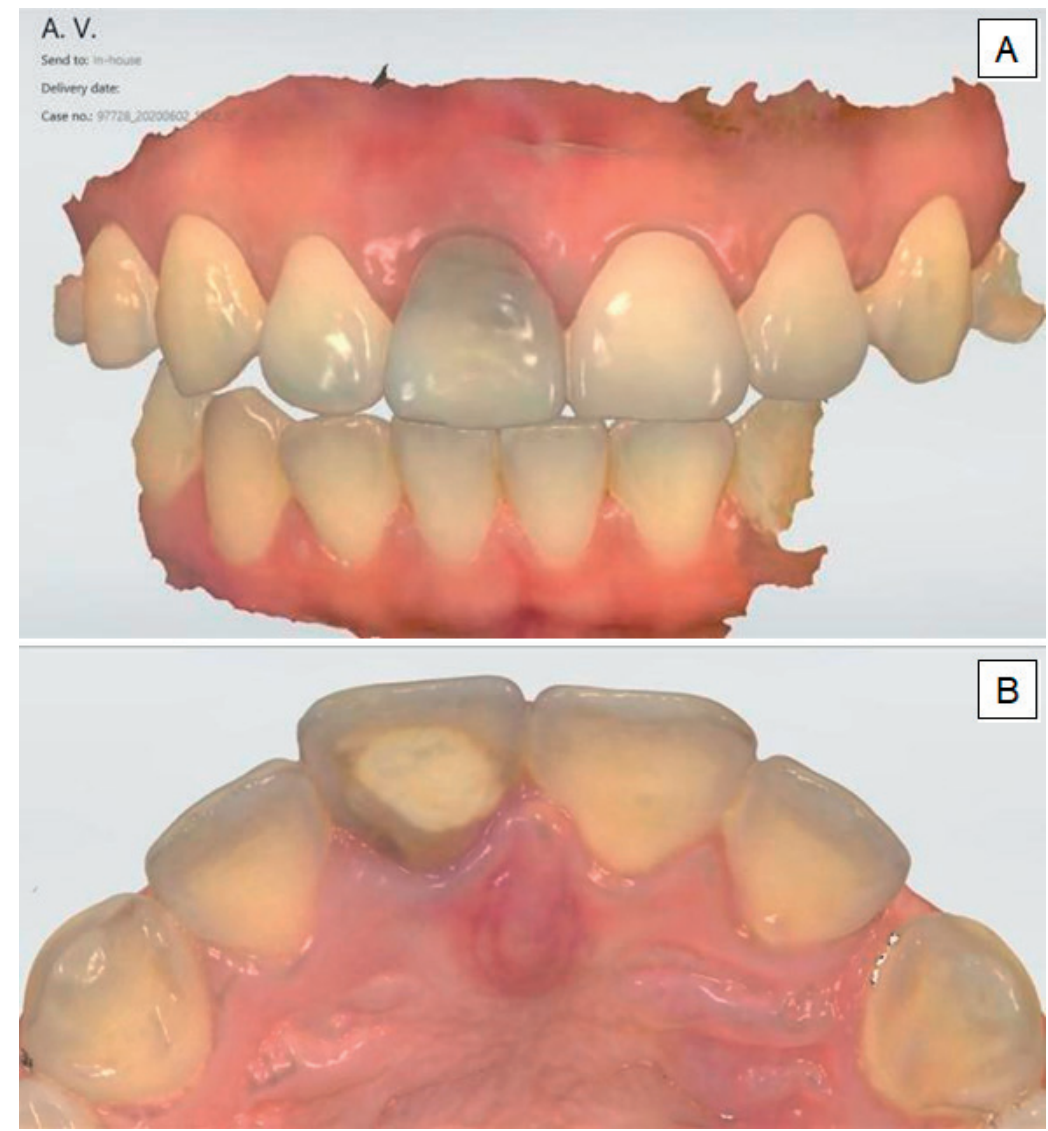

Figura 1. A. Imagen intraoral frontal que muestra un cambio de color severo en el incisivo central superior derecho. B. Vista oclusal.
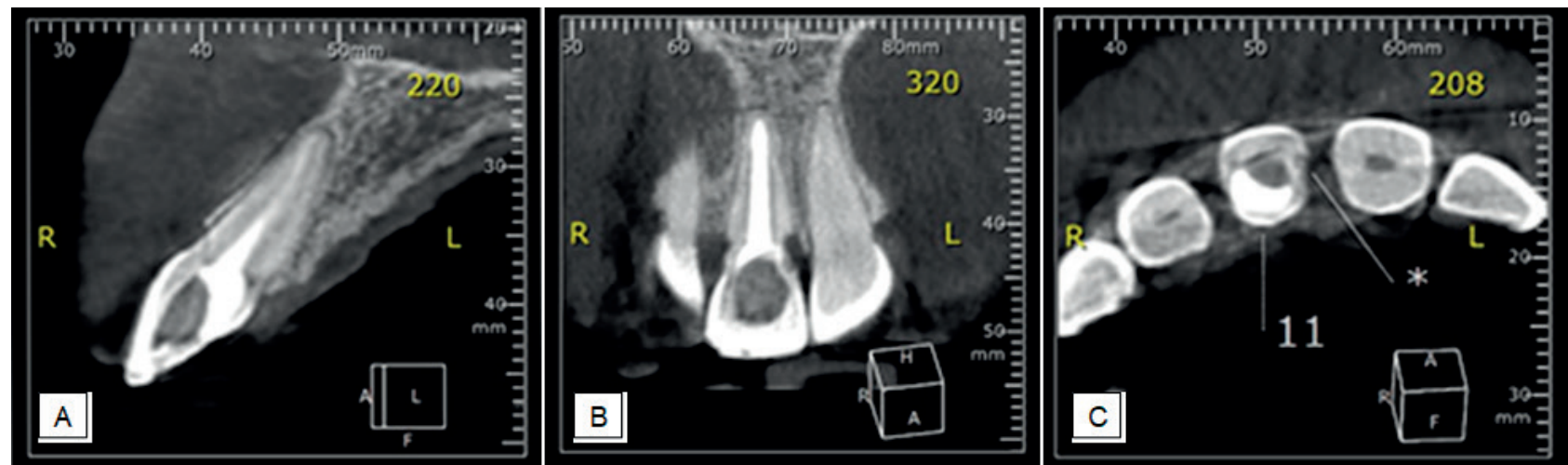

Figura 2. A. Tomografía del perímetro radicular conservado, región peri-radicular normal y presencia de línea de continuidad compatible con fractura. B y C. presencia de zona cervical hipodensa $\left({ }^{*}\right)$ en perímetro mesial asociada a modificación de contorno compatible con reabsorción

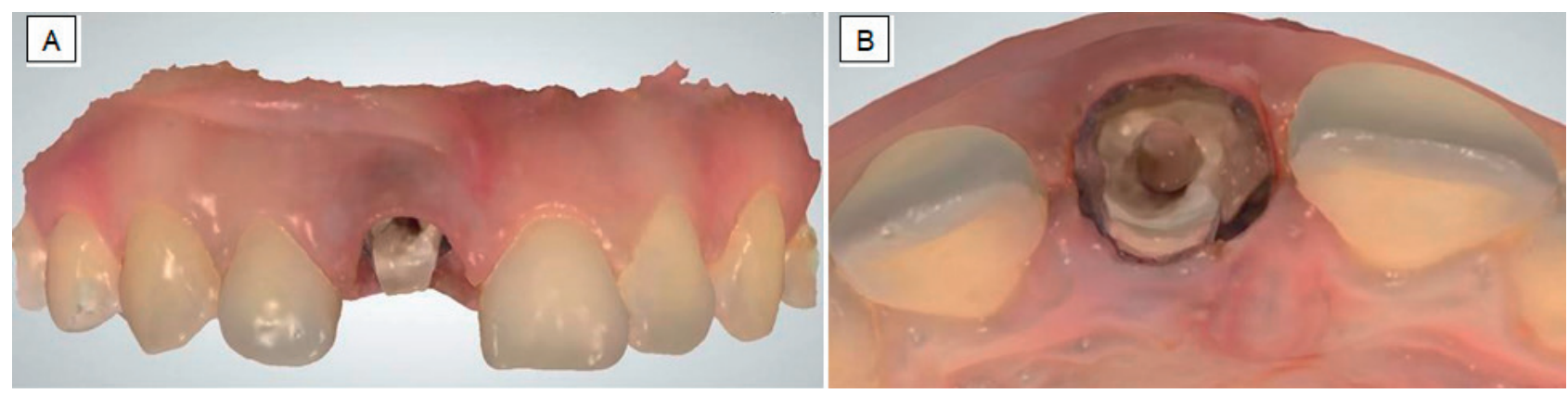

Figura 3. Imágenes de escáner intraoral del incisivo central superior derecho. A. Imagen frontal que muestra la estructura restante del diente. B. Áreas de reabsorción radicular externa en las regiones mesial y distal 


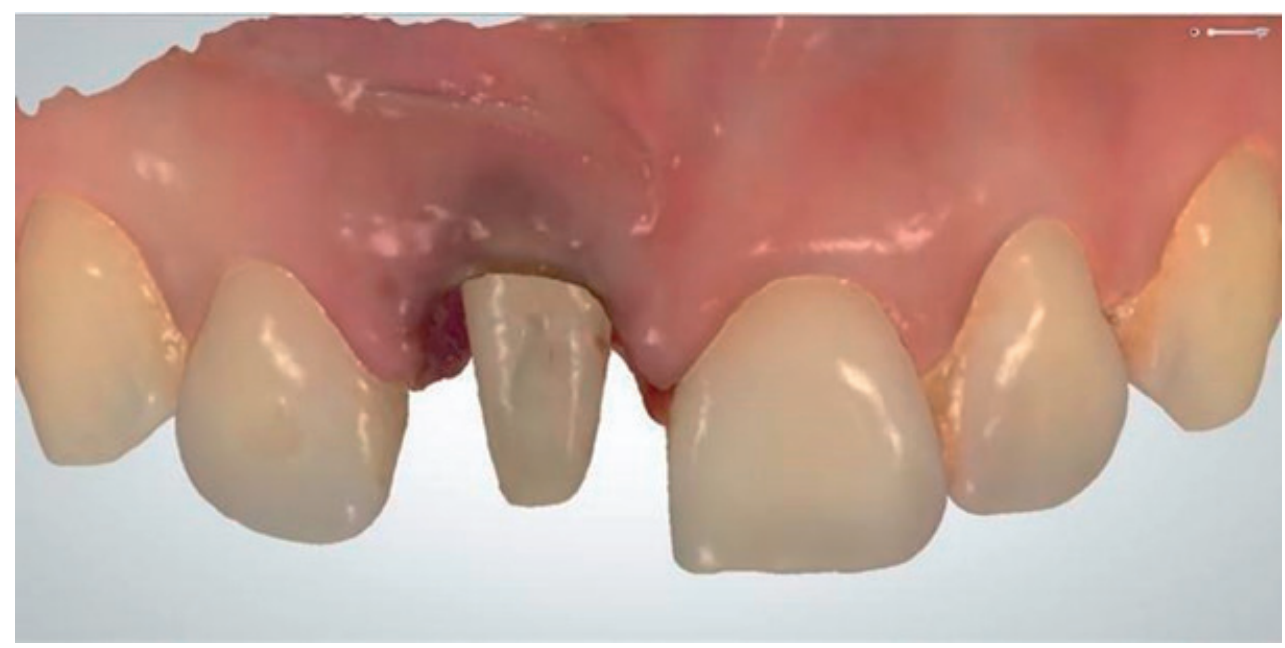

Figura 4. Imagen del escáner intraoral frontal que muestra la estructura dentaria restante del incisivo central superior derecho preparado según el concepto BOPT después de la instalación del poste de fibra de vidrio
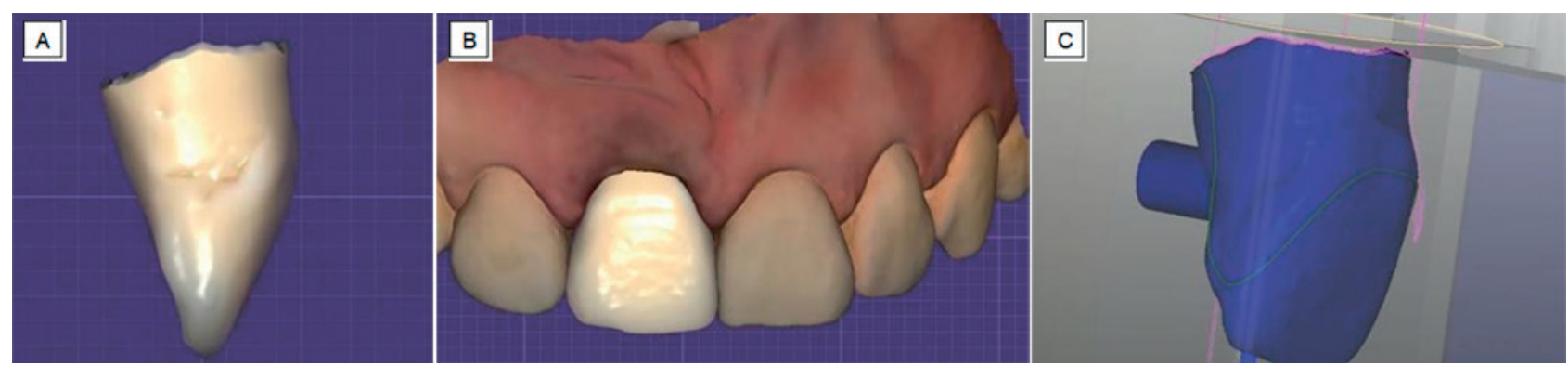

Figura 5. Imágenes digitales mostrando el diseño de la corona del incisivo central superior derecho usando una copia digital del diente original previamente escaneado. A. Corona de diente digitalizado. B. Corona del diente proyectado en el software Exocad. C. Archivo STL importado para ser fresado en la máquina CAM

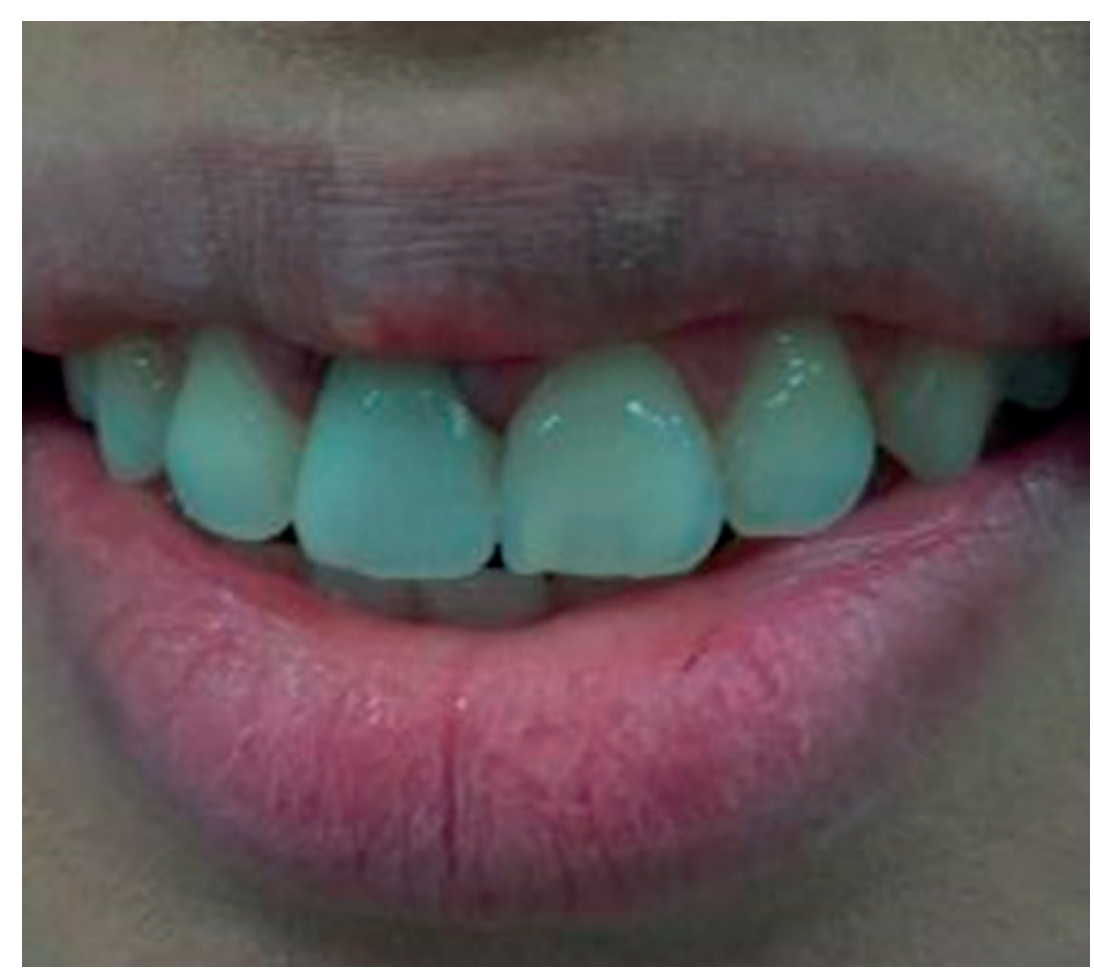

Figure 6. Imagen extraoral de la sonrisa del paciente después del procedimiento restaurador. La fotografía fue tomada por el mismo paciente al llegar a su casa. 
La cementación adhesiva de la restauración se realizó con cemento resinoso dual (Variolink Esthetic, Ivoclar Vivadent, Liechtenstein) siguiendo las instrucciones del fabricante, siendo fotopolimerizado finalmente (VALO LED Curing Light, Ultradent Products Inc). Se instruyó al paciente sobre el pronóstico y el carácter provisional del tratamiento realizado. Se programó una nueva cita para evaluar la salud de los tejidos duros y blandos y realizar la prótesis definitiva.

\section{Discusión}

En todo el mundo, varios centros de atención dental se limitaron a la atención de urgencia y emergencia ${ }^{7}$. Además, el miedo de las personas a la COVID-19 las hace reacias a acudir a lugares públicos y privados, incluidos hospitales médicos y dentales ${ }^{10}$. Sin embargo, el impacto psicosocial de la estética dental juega un papel importante en la búsqueda del tratamiento dental ${ }^{27}$. El trauma dental, una condición que afecta la estética facial, es una condición urgente que necesita ser tratada ${ }^{7,8}$.

Las restauraciones dentales en dientes anteriores suelen implicar procedimientos difíciles y que requieren mucho tiempo ${ }^{28}$. El uso de un flujo de trabajo digital puede ser una alternativa para ahorrar tiempo clínico ${ }^{28}$. El desarrollo de escáneres intraorales y sistemas CAD/CAM ha mejorado el flujo de trabajo digital de los tratamientos restauradores ${ }^{29}$. En este informe, se utilizó un abordaje digital con un escáner intraoral y un sistema CAD/ CAM como alternativa para restaurar un diente anterior traumatizado durante la pandemia de COVID-19. Esto nos permitió realizar el procedimiento en menos tiempo y, por lo tanto, limitar el riesgo de infección para el odontólogo y el paciente ${ }^{21}$.

Como se observa en el caso clínico, la reabsorción cervical externa suele ocurrir tras blanqueamiento intracoronal y traumatismo dental ${ }^{3,30-32}$. En este informe, la línea de fractura del diente estaba muy por debajo del margen gingival. Por lo tanto, se trata de un caso límite entre la extracción dentaria y la colocación de un implante dental o la rehabilitación mediante una restauración post-retenida ${ }^{33,34}$. Elegimos preservar la estructura dental restante. El concepto BOPT permite recrear la estética y el funcionamiento de una forma más sencilla y predecible, conservando la estructura dental remanente y creando una arquitectura estética gingival ideal ${ }^{25}$.

El papel de los profesionales de la odontología en la prevención de la transmisión de la COVID-19 es extremadamente importante ${ }^{9}$. El equipo odontológico siguió estrictamente las recomendaciones específicas para la práctica odontológica, incluido el cribado, las estrategias de control de infecciones y el manejo del paciente $5,6,8,11$. Antes del procedimiento, se le pidió a la paciente que se cepillara los dientes en una habitación adecuada. Además, se administró enjuague bucal con gluconato de clorhexidina al $0,12 \%$ durante 30 segundos para reducir la carga viral en la boca ${ }^{2,6,35}$. Aunque estas prácticas aún son confusas, este enfoque se utilizó para reducir la probabilidad de infección entre los profesionales y la contaminación ambiental ${ }^{36}$.
Se prefirieron las imágenes de TC a la radiografía intraoral ${ }^{6,8,11}$. Aunque el papel de la saliva en la infección por COVID-19 no está completamente explicado ${ }^{37}$, las radiografías intraorales pueden estimular la secreción de saliva y la tos, lo que puede maximizar el riesgo de infección ${ }^{6,14,38}$. Existe la posibilidad de transmisión de COVID-19 a través de aerosol 5,39,40. Por lo tanto, se utilizó el aislamiento con dique de goma del campo operatorio para minimizar la formación de gotas y aerosoles durante los procedimientos de restauración ${ }^{6}$.

El SARS-CoV-2 tiene una vida media de cinco a siete horas en superficies de plástico y acero inoxidable ${ }^{40}$. Los teléfonos móviles presentan un riesgo de transmisión de una variedad de microorganismos ${ }^{41,42}$. En nuestra práctica clínica, recomendamos encarecidamente que los pacientes y el personal dental desinfecten o no utilicen teléfonos móviles o cámaras en entornos dentales. Para obtener las imágenes del caso, optamos por utilizar un escáner intraoral como alternativa a las cámaras digitales ${ }^{43-45}$.

En la consulta se eligió el enfoque digital porque el tiempo de servicio se reduce significativamente ${ }^{17,19,20,46}$. Esto podría contribuir a reducir el riesgo de infección por COVID-19 en pacientes y dentistas ${ }^{21}$. Además, los sistemas digitales tienen la ventaja de ser más cómodos para el paciente, permitir una vista previa en 3D de las preparaciones dentales, y con potencial costo y efectividad en el tiempo ${ }^{47}$. En conjunto, esto es especialmente importante en el escenario actual de la pandemia de COVID-19.

En el presente caso clínico, el pronóstico con dos áreas de reabsorción externa y márgenes profundos de la preparación restauradora es dudoso. En este sentido, optamos por realizar un tratamiento temporal utilizando el flujo digital debido a la necesidad de un tratamiento inmediato por parte del paciente en el contexto de la pandemia de COVID-19. Así, este flujo de trabajo permitiría evitar la intervención directa de un laboratorio en la realización de las piezas definitivas. En las visitas de control y seguimiento, se puede considerar un plan restaurador con un pronóstico más predecible.

Con este caso se puede concluir la importancia de los enfoques digitales en odontología, incluidos los escáneres intraorales y los sistemas CAD/CAM, juegan un papel importante en la odontología. Esto podría contribuir a reducir el riesgo de infección por COVID-19 en pacientes y dentistas durante los tratamientos restauradores.

\section{Agradecimiento}

Queremos agradecer al Dr. Antônio Sergio Pereira Freitas por su asistencia técnica en la adquisición y análisis de la tomografía computarizada.

\section{Referencias bibliográficas}

1. Spagnuolo G, De Vito D, Rengo S, Tatullo M. COVID-19 outbreak: an overview on dentistry. Int J Environ Res Public Health. 2020;17(6):2094. DOI: 10.3390/ijerph17062094. 
2. Peng X, Xu X, Li Y, Cheng L, Zhou X, Ren B. Transmission routes of 2019-nCoV and controls in dental practice. Inter J Oral Sci. 2020;12(1):1-6. DOI: 10.1038/ s41368-020-0075-9

3. He F, Deng Y, Li W. Coronavirus disease 2019: What we know? J Med Virol. 2020;92(7):719-25. DOI: 10.1002/ jmv. 25766 .

4. Sim MR. The COVID-19 pandemic: major risks to healthcare and other workers on the front line. Occup Environ Med. 2020;77(5):281-2. DOI: 10.1136/oemed-2020-106567.

5. Ather A, Patel B, Ruparel NB, Diogenes A, Hargreaves KM. Coronavirus Disease 19 (COVID-19): Implications for Clinical Dental Care. J Endod. 2020;46(5):584-95. DOI: $10.1016 /$ j.joen.2020.03.008.

6. Jamal M, Shah M, Almarzooqi SH, Aber H, Khawaja S, El Abed R, et al. Overview of transnational recommendations for COVID-19 transmission control in dental care settings. Oral Dis. 2021;27(Suppl 3):655-64. DOI: 10.1111/odi.13431.

7. Alharbi A, Alharbi S, Alqaidi S. Guidelines for dental care provision during the COVID-19 pandemic. S Dent J. 2020;32(4):181-6. DOI: 10.1016/j.sdentj.2020.04.001.

8. Lucaciu O, Tarczali D, Petrescu N. J Dent Sci. 2020;15(4):399-402. DOI: 10.1016/j.jds.2020.04.012.

9. Coulthard P. Dentistry and coronavirus (COVID-19)-moral decision-making. $\mathrm{Br}$ Dent J. 2020;228(7):503-505. DOI: 10.1038/s41415-0201482-1.

10. Guo H, Zhou Y, Liu X, Tan J. The impact of the COVID-19 epidemic on the utilization of emergency dental services. J Dent Sci. 2020;15(4):564-7. DOI: 10.1016/j. jds.2020.02.002.

11. Recomendações A, para atendimento odontológico COVID C. Comitê de Odontologia AMIB/CFO de enfrentamento ao COVID-19 Departamento de Odontologia AMIB-1 Atualização 25/03/2020. Disponible en: https://www.amib.org.br/fileadmin/ user_upload/amib/2020/junho/22/220620Recomendacoes_AMIB_-_CFO_para_atendimento_odontologico_COVID-19.pdf.

12. BRASIL. NOTA TÉCNICA GVIMS/GGTES/ANVISA No 04/2020. In: Sanitária ANdV, editor. Orientaçóes para Serviços de Saúde: Medidas de Prevenção e Controle que devem ser adotadas durante a assistência aos casos suspeitos ou confirmados de infecção pelo novo Coronavírus (SARS-COV-2). Brasília: ANVISA; 2020. Disponible en: https://www.gov.br/anvisa/pt-br/centraisdeconteudo/publicacoes/servicosdesaude/notas-tecnicas/nota-tecnica-gvims_ggtes_anvisa-04_2020-25-02para-o-site.pdf.

13. Gomes RL, Pedrosa MS, Silva CHV. Restorative dental treatment in times of COVID-19. Rev Gaúch Odontol. 2020;68:e20200019. DOI: 10.1590/1981863720200001920200075 .

14. Meng L, Hua F, Bian Z. Coronavirus disease 2019 (COVID-19): emerging and future challenges for dental and oral medicine. J Dent Res. 2020;99(5):481-7. DOI: 10.1177/0022034520914246.
15. Lo Russo L, Caradonna G, Biancardino M, De Lillo A, Troiano G, Guida L. Digital versus conventional workflow for the fabrication of multiunit fixed prostheses: A systematic review and meta-analysis of vertical marginal fit in controlled in vitro studies. J Prosthet Dent. 2019;122(5):435-40. DOI: $10.1016 / j$. prosdent.2018.12.001.

16. Mangano F, Veronesi G. Digital versus Analog Procedures for the Prosthetic Restoration of Single Implants: A Randomized Controlled Trial with 1 Year of Follow-Up. BioMed Res Int. 2018;2018:5325032. DOI: $10.1155 / 2018 / 5325032$.

17. Joda T, Zarone F, Ferrari M. The complete digital workflow in fixed prosthodontics: a systematic review. BMC Oral Health. 2017;17(1):124. DOI: 10.1186/s12903017-0415-0.

18. Li J, Chen Z, Dong B, Wang HL, Yu H. A digital workflow with computer-assisted implant planning for fabricating an impression splinting framework and custom tray for multiple implants. J Prosthet Dent. 2020;124(3):262269. DOI: $10.1016 /$ j.prosdent.2019.08.021.

19. Mühlemann S, Benic GI, Fehmer V, Hämmerle CHF, Sailer I. Randomized controlled clinical trial of digital and conventional workflows for the fabrication of zirconia-ceramic posterior fixed partial dentures. Part II: Time efficiency of CAD-CAM versus conventional laboratory procedures. J Prosthet Dent. 2019;121(2):252-7. DOI: 10.1016/j.prosdent.2018.04.020.

20. Sailer I, Benic GI, Fehmer V, Hämmerle CHF, Mühlemann S. Randomized controlled within-subject evaluation of digital and conventional workflows for the fabrication of lithium disilicate single crowns. Part II: CAD-CAM versus conventional laboratory procedures. J Prosthet Dent. 2017;118(1):43-8. DOI: 10.1016/j. prosdent.2016.09.031.

21. Papi P, Di Murro B, Penna D, Pompa G. Digital prosthetic workflow during COVID-19 pandemic to limit infection risk in dental practice. Oral Dis. 2021;27(Suppl 3):723-6. DOI: 10.1111/odi.13442.

22. Yeung S, Parminter P, Goto Y, Aghivinian C. A CAD-CAM approach for designing and fabricating a periodontal prosthesis: A clinical report. J Prosthet Dent. 2020;123(4):553-6. DOI: 10.1016/j.prosdent.2019.02.021.

23. Grandini S, Sapio S, Simonetti M. Use of anatomic post and core for reconstructing an endodontically treated tooth: a case report. J Adhes Dent [Internet]. 2003 [citado el 08 de agosto de 2021]; 5(3):243-7. Disponible en: https://pubmed.ncbi.nlm.nih.gov/14621246/.

24. Ok E, Erdek Y. Re-attachment of subgingivally oblique fractured central incisor using a fiber post. Eur J Dent [Internet]. 2008 [citado el 08 de agosto de 2021]; 2(02):138-41. Disponible en: https://pubmed.ncbi.nlm. nih.gov/19212525/

25. Loi I, Di Felice A. Biologically oriented preparation technique (BOPT): a new approach for prosthetic restoration of periodontically healthy teeth. Eur J Esthetic Dent [Internet]. 2013 [citado el 08 de agosto de 2021]; 8(1):10-23. Disponible en: https://pubmed.ncbi.nlm. nih.gov/23390618/. 
26. Rocha AT, Gonçalves LM, Vasconcelos A JdC, Maia Filho M, Nunes Carvalho C, De Jesus Tavarez RR. Effect of anatomical customization of the fiber post on the bond strength of a self-adhesive resin cement. Int J Dent. 2017;2017:5010712. DOI: 10.1155/2017/5010712.

27. Lin F, Ren M, Yao L, He Y, Guo J, Ye Q. Psychosocial impact of dental esthetics regulates motivation to seek orthodontic treatment. Am J Orthod Dentofacial Orthop. 2016;150(3):476-82. DOI: 10.1016/j.ajodo.2016.02.024.

28. Coachman C, De Arbeloa L, Mahn G, Sulaiman T, Mahn E. An Improved Direct Injection Technique with Flowable Composites. A Digital Workflow Case Report. Oper Dent. 2020;45(3):235-42. DOI: 10.2341/18-151T.

29. Revilla-León M, Besné-Torre A, Sánchez-Rubio JL, Fábrega JJ, Özcan M. Digital tools and 3D printing technologies integrated into the workflow of restorative treatment: A clinical report. J Prosthet Dent. 2019;121(1):3-8. DOI: 10.1016/j.prosdent.2018.02.020.

30. Heithersay GS. Invasive cervical resorption following trauma. Aust Endod J. 1999;25(2):79-85. DOI: 10.1111/j.1747-4477.1999.tb00094.x.

31. Patel S, Kanagasingam S, Ford TP. External cervical resorption: a review. J Endod. 2009;35(5):616-25. DOI: 10.1016/j.joen.2009.01.015.

32. Goon WW, Cohen S, Borer RF. External cervical root resorption following bleaching. J Endod. 1986;12(9):4148. DOI: 10.1016/S0099-2399(86)80076-0.

33. Portera JJ, Thomson JA. Reuse of existing crown after tooth fracture at the gingival margin. J Prosthet Dent. 1983;50(2):195-7. DOI: 10.1016/00223913(83)90011-2.

34. Iqbal MK, Kim S. For teeth requiring endodontic treatment, what are the differences in outcomes of restored endodontically treated teeth compared to implant-supported restorations?. Int J Oral Maxillofac Implants [Internet]. 2007 [citado el 08 de Agosto de 2021]; 22(Suppl):96-116. Disponible en: https://pubmed.ncbi. nlm.nih.gov/18437793/

35. Eduardo FdP, Corrêa L, Heller D, Daep CA, Benitez C, Malheiros Z, et al. Salivary SARS-CoV-2 load reduction with mouthwash use: A randomized pilot clinical trial. Heliyon. 2021;7(6):e07346. DOI: 0.1016/j.heliyon.2021.e07346.

36. Ortega KL, Camargo ARd, Franco JB, Azul AM, Sayáns MP, Silva PHB. SARS-CoV-2 and dentistry. Clin Oral Investig. 2020;24:2541-2542. DOI: 10.1007/s00784020-03381-7.

37. Pedrosa M, Sipert CR, Nogueira FN. Salivary Glands, Saliva and Oral Presentations in COVID-19 infection. Pesqui Bras Odontopediatria Clin Int. 2020;20(suppl 1):e0104. DOI: 10.1590/pboci.2020.112.
38. Vandenberghe B, Jacobs R, Bosmans H. Modern dental imaging: a review of the current technology and clinical applications in dental practice. European radiology. 2010;20(11):2637-55. DOI: 10.1007/s00330-0101836-1.

39. Ge Zy, Yang Lm, Xia Jj, Fu Xh, Zhang Yz. Possible aerosol transmission of COVID-19 and special precautions in dentistry. J Zhejiang Univ Sci B. 2020;21(5):361-8. DOI: 10.1631 /jzus.B2010010.

40. Van Doremalen N, Bushmaker T, Morris DH, Holbrook MG, Gamble A, Williamson BN, et al. Aerosol and surface stability of SARS-CoV-2 as compared with SARSCoV-1. N Engl J Med. 2020;382(16):1564-7. DOI: 10.1056/NEJMc2004973.

41. Heyba M, Ismaiel M, Alotaibi A, Mahmoud M, Baqer H, Safar A, et al. Microbiological contamination of mobile phones of clinicians in intensive care units and neonatal care units in public hospitals in Kuwait. BMC infectious diseases. 2015;15:434. DOI: 10.1186/ s12879-015-1172-9.

42. Qureshi NQ, Mufarrih SH, Irfan S, Rashid RH, Zubairi AJ, Sadruddin A, et al. Mobile phones in the orthopedic operating room: Microbial colonization and antimicrobial resistance. World J Orthop. 2020;11(5):252-64. DOI: 10.5312/wjo.v11.i5.252.

43. Mangano F, Gandolfi A, Luongo G, Logozzo S. Intraoral scanners in dentistry: a review of the current literature. BMC Oral Health. 2017;17(1):149. DOI: 10.1186/ s12903-017-0442-x.

44. Sfondrini MF, Gandini P, Malfatto M, Di Corato F, Trovati F, Scribante A. Computerized casts for orthodontic purpose using powder-free intraoral scanners: accuracy, execution time, and patient feedback. Biomed Res Int. 2018;2018:4103232. DOI: 10.1155/2018/4103232.

45. Reyes J, Acosta P, Ventura D. Repeatability of the human eye compared to an intraoral scanner in dental shade matching. Heliyon. 2019;5(7):e02100. DOI: 10.1016/j. heliyon.2019.e02100.

46. Joda T, Brägger U. Time-efficiency analysis of the treatment with monolithic implant crowns in a digital workflow: a randomized controlled trial. Clin Oral Implants Res. 2016;27(11):1401-6. DOI: 10.1111/clr.12753.

47. Yuzbasioglu E, Kurt H, Turunc R, Bilir H. Comparison of digital and conventional impression techniques: evaluation of patients' perception, treatment comfort, effectiveness and clinical outcomes. BMC Oral Health. 2014;14:10. DOI: 10.1186/1472-6831-14-10. 